\title{
Reseña de los estudios sobre el Vaticano II de Estudio Agustiniano (1966-2012)
}

\author{
RAMÓN SALA, OSA*
}

RESUMEN: Los temas tratados por el Concilio Vaticano II han sido una referencia de la revista Estudio Agustiniano desde su fundación (1966). En estas líneas se pasa revista a los artículos publicados en ella que tienen por objeto específico la doctrina conciliar.

PALABRAS CLAVE: Estudio Agustiniano - Vaticano II.

ABSTRACT: The topics related to the Council Vatican II have been a reference for the review Estudio Agustiniano since its foundation (1966). Here there is an overview of those articles edited by it that have the council doctrines as its specific subject.

KEY WORDS: Estudio Agustiniano - Vatican II.

Como tantas otras publicaciones teológicas semejantes, la revista Estudio Agustiniano $(E s t A g)^{1}$ es también un fruto de primera hora del acontecimiento conciliar. En efecto, su primer número apareció en 1966, sólo un año después de la clausura del Concilio Vaticano II. La nueva revista se anticipaba, también en un año, a la erección del "Estudio Teológico Vallisoletano de la Orden de San Agustín” como centro afiliado-

* Profesor del Estudio Teológico Agustiniano (Valladolid). rssala@hotmail.com

1 Es la abreviatura del catálogo oficial IATG (Internationales Abkürzungsverzeichnis für Theologie und Grenzgebiete). Cf. S. M. ScHWERTNER, Zg., Theologische Realenzyclopädie (TRE). Abkürzungsverzeichnis. 2. überarbeitete und erwertete Auflage, Berlin-New York 1994, p. 53. 
a la Universidad Pontificia de Comillas (22 de mayo de 1967). Una iniciativa dirigida a elevar el nivel de los estudios eclesiásticos conforme a las exigencias del Concilio Vaticano $\mathrm{II}^{2}$.

Esta revista cuatrimestral -actualmente con una tirada de unos cuatrocientos ejemplares- se recibe en los principales centros teológicos nacionales e internacionales y mantiene una relación de intercambio con unas doscientas cincuenta revistas especializadas de carácter filosófico y teológico. Ha tenido dos épocas. La primera -vols. 1-45- comprende desde su fundación hasta el año 2010. La segunda época se ha iniciado en el 2011 y presenta como principales innovaciones un formato externo renovado $y$ la constitución de un Comité Científico internacional integrado por catorce profesores de reconocido prestigio.

Hasta el presente número han aparecido en EstAg un total de dieciséis trabajos -entre artículos y "textos y glosas"- que tratan directa y expresamente de la temática del Concilio Vaticano II. La presente reseña se limita a dar cuenta solamente de esos estudios. Por consiguiente, no se abordan otros que pudieran contener referencias indirectas a los textos conciliares, en el marco de otras temáticas diversas o más amplias. Ha sido preciso acotar de este modo el objeto de estudio, pues resultan inevitables las citas de los documentos del Vaticano II en cualquier ensayo teológico postconciliar de carácter científico.

Una última premisa antes de entrar en materia. Se pueden distinguir claramente tres períodos respecto a la atención prestada por EstAg al Concilio Vaticano II. El más productivo es el primero. Las dos terceras partes del total de los estudios que vamos a presentar corresponden a la segunda mitad de los años 60 y a los años 70 . Es decir, se trata de artículos publicados en el inmediato postconcilio, que reflejan el impacto inicial de unos documentos que no sólo resultaban novedosos, sino especialmente atractivos por su talante renovador. Sorprendentemente, en una segunda etapa, nos encontramos con una presencia puramente testimonial -sólo dos artículos- de la recepción del Concilio desde la perspectiva de sus primeros 20 ó 25 años. Por último, tras el paréntesis en blanco de los años 90 , con el s. XXI ha reaparecido paulatinamente en la revista el interés por el Vaticano II.

Para la exposición se han clasificado los estudios publicados por la revista en tres grupos: trabajos de conjunto o temáticos sobre el Vaticano II en

2 Cf. J. ÁlvareZ, "Estudio Teológico Agustiniano de Valladolid. XXV aniversario": EstAg 27 (1992) 551-594; I. RodRígueZ-J. ÁlvareZ, Al servicio del evangelio. Provincia Agustiniana del Santísimo Nombre de Jesús de Filipinas, Valladolid 1996, p. 359. 
general, estudios particulares sobre la Constitución pastoral Gaudium et spes (GS) ${ }^{3}$ y ensayos que abordan otros documentos conciliares.

\section{ESTUDIOS SOBRE EL VATICANO II}

En las páginas de EstAg podemos encontrar una pluralidad de aproximaciones a la temática del Concilio Vaticano II. Hay un estudio de carácter pastoral que, tomando la doctrina agustiniana como "telón de fondo histórico", trata de la cooperación de los religiosos en la pastoral diocesana (Fernando Campo) a la luz, sobre todo, del decreto CD sobre el ministerio de los obispos ${ }^{4}$. Desde el punto de vista de la espiritualidad del Vaticano II tenemos dos estudios. El primero (Carlos González) está enfocado a partir de la Constitución dogmática LG, y en concreto de LG 31. Presenta sucesivamente la espiritualidad laical (cf. GS 43), la espiritualidad religiosa (cf. LG 43-44) y la espiritualidad sacerdotal (cf. PO 3-4) ${ }^{5}$. El segundo artículo se centra en el tratamiento que los documentos del Concilio (cf. LG 12; GS 21; AA 3-4) dan a las virtudes teologales ${ }^{6}$. Según la doctrina del Vaticano II es posible superar el dualismo entre una vida cristiana "teologal" y la secularidad del mundo. Para el autor (Jesús Fernández), la "tarea más urgente" le espera a la caridad: "convencer a todos de que el mundo hay que transfigurarlo por la fuerza de las bienaventuranzas...".

Hay un único trabajo que aborda la temática del Concilio desde la perspectiva bíblica. Su autor, Constantino Mielgo, profesor de Sagrada Escritura del Estudio Teológico de Valladolid (1968-2008), estudia el uso de la Biblia por parte de los textos del Vaticano II. En concreto, su artículo analiza la lectura conciliar del $A T^{7}$. El autor comienza situando el estudio dentro de la problemática de la relación entre los dos Testamentos, un tema

${ }^{3}$ Entre los documentos del Concilio GS es, con mucha diferencia, el documento al que los autores han prestado una mayor atención en EstAg (seis textos). En este artículo se citan los documentos del Concilio Vaticano II empleando las abreviaturas comunes.

4 Cf. F. CAMPo DEL Pozo, "Pastoral de conjunto de clérigos y religiosos según san Agustín y el concilio Vaticano II: EstAg 11 (1976) 473-498 (propiamente sobre la doctrina conciliar en pp. 481-489).

${ }^{5}$ Cf. C. GonZÁleZ SÁNCHEZ, "Espiritualidad seglar, religiosa y sacerdotal a la luz del Concilio": EstAg 4 (1969) 567-576.

6 Cf. J. FernándeZ, "Teología y secularización de las virtudes en el Vaticano II": EstAg 5 (1970) 533-562.

7 C. Mielgo Fernández, "La difícil lectura del Antiguo Testamento hecha por el Concilio Vaticano II": EstAg 21 (1986) 351-365. 
"complicado y que el concilio no resuelve". Pero limita su exposición a tratar de explicar la interpretación -tanto teórica, como práctica- que, según el Vaticano II, debe hacerse del primer Testamento. El artículo consta de tres partes. A partir del principio fundamental, contenido en DV 12, y siguiendo el cap. IV de la Constitución dogmática sobre la Revelación, las dos primeras partes del estudio afrontan el aspecto teórico de la cuestión. Se repasan sucesivamente la "doctrina sobre el sentido literal" y la "doctrina sobre el AT" contenidas en DV. Según ese documento conciliar, en la "lectura cristiana" de los textos bíblicos hay que respetar siempre la intención original y el sentido que los autores quisieron dar a sus escritos. En la última parte del estudio, titulada "Uso que hace el concilio del AT", se trata el aspecto práctico del tema. El autor resalta, ante todo, la "sobriedad" con que los documentos del Concilio citan el $\mathrm{AT}^{8}$. El Vaticano II emplea correctamente el AT cuando habla de un modo genérico del hombre o de Dios y el mundo (cf. GS 12; GS 57; etc.). Es decir, cuando aborda cuestiones de antropología teológica. En cambio, la lectura de los textos veterotestamentarios (cf. Jer 31,31-34; Gn 3,15; Is 11,12; etc.) presenta muchas más dificultades cuando se hacen afirmaciones cristológicas, mariológicas o eclesiológicas $^{9}$. El autor concluye poniendo de relieve la contradicción entre la teoría y la práctica en la lectura que hace del AT el Vaticano II.

A continuación pasamos revista a tres trabajos de carácter sistemático. Los dos primeros son estudios de conjunto del Vaticano II y tienen como autores a otros dos profesores del Estudio Teológico. El tercero, el último aparecido cronológicamente (2006), es más breve y trata una temtica particular.

El "Cristocentrismo" del Concilio Vaticano II es el tema de uno de los artículos de José Morán ${ }^{10}$, uno de los profesores del Estudio (1967-1970) cuyas colaboraciones aparecen con más frecuencia en las páginas de los primeros volúmenes de EstAg. La exposición consta de tres partes. La primera, "Cristo revelador" contiene un comentario a DV 2-3. La segunda, "Cristo y la Iglesia", analiza los primeros capítulos de LG (nn. 1-10) y reflexiona sobre la presencia de Cristo en la liturgia de la Iglesia (SC 7). La última parte, "Cristo y el hombre", estudia los números conclusivos

\footnotetext{
8 Apenas 87 referencias (de las cuales sólo 18 son citas explícitas), frente a las aproximadamente 1300 del NT (cf. Ib., 360).

9 Cf. Ibid., 360-363.

10 J. MoRÁn FernándeZ, "Cristo y el cristocentrismo en el Concilio Vaticano II": EstAg 3 (1968) 527-543. Más adelante se hace referencia a otro artículo anterior del mismo autor sobre la Constitución pastoral del Concilio Vaticano II (cf. ID., "El hombre y los valores humanos en el Concilio Vaticano II": EstAg 1 (1966) 87-114).
} 
-estratégicamente cristológicos- de la exposición preliminar (GS 10) y de cada uno de los capítulos de la primera parte de la Constitución pastoral (cf. GS 22; 32; 39; 45). Aquí se comentan también algunos textos de los decretos sobre la actividad misionera (cf. AG 3, 8 y 12) y sobre el apostolado seglar (AA 8). En el Vaticano II, la Iglesia, que continúa la obra de Cristo en el mundo, ha colocado a Cristo en el centro de la historia, presentándolo como necesario punto de referencia en la solución de los problemas humanos.

Con el título intencionadamente abierto de Alrededor del Vaticano $I I^{11}$, el ensayo del profesor Tomás Marcos, docente de dogmática en el Estudio de Valladolid, tiene un doble objetivo. En primer lugar, indagar en las causas que condujeron a la "revolución eclesial" que representó el Concilio Vaticano II. Y, después, mostrar las "expectativas y consecuencias" que se derivan de la asamblea conciliar. Corresponden a las dos secciones en que está organizado el texto. Entre los factores que contribuyeron a impulsar la celebración del Concilio, el autor distingue por un lado, lo que llama "envites sociales" (mentalidad racionalista y secular, efervescencia de la individualidad); y por otro, los movimientos eclesiales (promoción de lo secular y aceptación de métodos científicos por la teología). En la segunda parte del trabajo, "El tiempo de después", el autor analiza cuatro cuestiones. Primero: la recepción del Concilio, elencando las diversas tomas de postura ante él. Entre la aceptación mayoritaria y el rechazo minoritario, también se alude a la postura ecléctica de los que, asumiendo el Vaticano II, manifiestan a la vez desconfianza hacia sus resultados. Segundo: desde el punto de vista de la eclesiología y como uno de los efectos del Concilio, la participación personal debido a cierto cambio en la conciencia de pertenencia a la Iglesia ("Iglesia somos todos") 12 . Tercero: el tema de la democratización de las estructuras eclesiales y la importancia de la colegialidad episcopal a raíz del Concilio. El autor advierte de que "tal vez es la reforma en la que el postconcilio menos ha avanzado". Finalmente, se trata la problemática de la situación de la mujer -“Con la mujer hemos topado"- en la Iglesia ${ }^{13}$. Después del Vaticano II es totalmente inaceptable su multisecular discriminación o sumisión. El artículo desacredita todo intento por justificarlas. Concluye el autor afirmando que todas aquellas cuestiones deben recibir una respues-

11 T. MARCos-MARTíneZ, “Alrededor del Vaticano II": EstAg 38 (2003) 589-610.

12 El autor desarrolla ampliamente esta temática en otro artículo anterior de esta misma revista (cf. "Laicos e Iglesia": EstAg 27 (1992) 523-550).

13 Cf. "Alrededor del Vaticano II", 605-610. 
ta en la línea de la renovación conciliar. De otro modo "el Vaticano II será sólo el grito de la imposible modernidad de la Iglesia".

El 23 de mayo de 2007, el Estudio Teológico de Valladolid organizó un acto académico en el marco de la celebración oficial de su cuadragésimo aniversario. Dicho evento contó con la intervención de diversas personalidades relacionadas con el centro. Entre ellas, la del profesor Santiago Madrigal, eclesiólogo y un experto en el Vaticano II, entonces decano de la Facultad de Teología de la Universidad Pontificia de Comillas. Su ponencia versó sobre el estudio de la teología a la luz del Vaticano II $^{14}$. El teólogo jesuita comenzaba su exposición haciendo referencia a la inesperada respuesta que Salvador Dalí daba a la pregunta acerca de su opinión sobre el Concilio Vaticano II, en el famoso libro de J. Ma . Gironella, Cien españoles y Dios (Barcelona 1969). - "Íntimamente no soy partidario del concilio, pero por razones propias de mi estructura jesuítica lo defiendo". En sus "reflexiones fragmentarias", S. Madrigal considera que, según la doctrina conciliar, el eje central de los estudios teológicos debe ser el "Mysterium Christi". Después de recordar el título de la Relación Final del Sínodo extraordinario de los Obispos sobre el Concilio Vaticano II (1985), que articulaba en una frase el tema de las cuatro Constituciones conciliares $^{15}$, afirma que todo plan de estudios que quiera ser fiel al Vaticano II debe necesariamente incorporar esos mismos postulados: la eclesialidad (LG), la centralidad de la Escritura (DV), la dimensión celebrativa (SC) y la salvación del mundo (GS). A continuación, el autor esboza, como en círculos concéntricos, los que -a su juicio-deberían ser los núcleos temáticos del ciclo institucional o primer ciclo de Teología: misterio de Dios manifestado en Cristo Jesús (Cristología-Trinidad), misterio de la Iglesia (Eclesiología), teología de los sacramentos (Sacramentología) y origen-destino del hombre (Antropología-Escatología). Tras una breve alusión a los métodos de estudio (cf. OT 13-18) y una referencia al agustino Lutero, el artículo culmina con la siguiente reflexión de sabor barthiano: "En teología no se trata primariamente de lo que se dice de Dios, ni siquiera de lo que la fe dice de Él, sino de lo que Él mismo dice de sí en el momento en que se manifiesta, y por tanto de Dios mismo".

14 S. MADRIGAL, "El Concilio Vaticano II y el estudio sistemático de la teología": EstAg 43 (2008) 507-510.

15 "Ecclesia sub Verbo Dei mysteria Christi celebrans pro salute mundi". 


\section{ESTUDIOS SOBRE LA CONSTITUCIÓN PASTORAL GS}

Ningún otro documento conciliar ha despertado tanto interés en la revista EstAg como GS. En el apartado anterior hemos comprobado cómo al tratar distintos temas en los documentos del Vaticano II, inevitablemente se cita a menudo GS. En este apartado se presentan de un modo diacrónico los estudios que abordan de una forma exclusiva la temática contenida en los textos de la Constitución pastoral.

Ya en el número inaugural de la revista, EstAg 1 (1966) encontramos dos trabajos sobre la Constitución pastoral GS. Tratan del ateísmo moderno y de los valores humanos, respectivamente. Dos temas, por cierto, con muchos puntos de contacto. El artículo del doctor Argimiro Turrado ${ }^{16}$, ilustre agustinólogo y profesor de dogmática en el Estudio Teológico (1967-1994), ofrece "algunas consideraciones sobre el ateísmo moderno y la teología cristiana, siguiendo de cerca las directrices del Concilio Vaticano II". El autor hace un análisis pormenorizado del fenómeno del ateísmo moderno, comentando sistemáticamente GS 19-21. Según él, el documento conciliar sobre la Iglesia en el mundo de hoy descubre también los aspectos positivos "al menos indirectamente" del ateísmo moderno ${ }^{17}$. En particular explica su contribución a purificar el concepto de Dios y a favorecer el humanismo. Después de presentar lo que denomina "mensaje agustiniano del Concilio Vaticano II, el artículo termina con una reflexión sobre "el humanismo ateo y el cristianismo". Por su parte, el profesor José Morán ofrece un estudio de carácter antropológico sobre los valores humanos ${ }^{18}$. Comienza preguntándose quién es el "hombre" del que habla el Concilio en GS. Para el autor, el Vaticano II no se refiere a un hombre "abstracto sin raigambre en la cuotidianidad [sic]", sino al "hombre existencial". Cuando habla del hombre, GS manifiesta "un optimismo radical", una gran simpatía por los logros del espíritu humano. Ahora bien, tampoco oculta los desequilibrios del mundo

16 A. Turrado Turrado, “Ateísmo moderno y Teología”: EstAg 1 (1966) 47-65.

17 Cf. Ib. 60-65.

18 J. MORÁN FERNÁNDEZ, "El hombre y los valores humanos en el Concilio Vaticano II": EstAg 1 (1966) 87-114. Además del artículo, ya reseñado, sobre el cristocentrismo del Vaticano II, este autor tiene también una colaboración cuyo título (“¿Un signo de los tiempos?": EstAg 5 (1970) 393-404) podría llevar a engaño (cf. GS 4a). No es un estudio sobre GS, sino la crónica de un ciclo de conferencias con motivo de la inauguración del Instituto Patrístico Augustinianum (Roma, 4-7 de mayo de 1970). 
(GS 8) a los que da lugar el pecado (GS 13)19 “ ¿Cuáles son los valores humanos?”, se pregunta el autor. Según GS, la dignidad de la persona humana compendia la totalidad de los valores humanos. Es una noción que resuena en muchas de las páginas de los documentos conciliares. La dignidad de la persona tiene como fundamento la condición del hombre como imago Dei y es la fuente de todos los demás valores tanto personales (igualdad, libertad, respeto), como sociales (convivencia fraterna, cooperación, diálogo). En la conclusión se afirma que ha nacido "un nuevo humanismo" (GS 55), que ha esparcido su voz desde el Vaticano II y que pretende "crear una vida más humana" a través de la cultura, la política, la promoción de la paz. Como recordaba Pablo VI en su Discurso de clausura del Vaticano II, se puede decir que en GS el Concilio enseña un modo nuevo y solemne de amar al hombre para amar a $\operatorname{Dios}^{20}$.

El primer capítulo de la segunda parte de GS, dedicado al "Matrimonio y la Familia" es objeto de estudio desde el punto de vista moral por parte del profesor Zacarías Herrero ${ }^{21}$, docente de Teología Moral en el Estudio Teológico de Valladolid (1967-1992) y en el Instituto Patrístico Augustinianum de Roma. El autor comienza reconociendo que el Concilio fue muy cauto a la hora de presentar soluciones prácticas sobre algunas cuestiones de moral matrimonial especialmente controvertidas (p.e. regulación de la natalidad). En cambio, en su artículo desarrolla cuatro puntos de la enseñanza de la Constitución pastoral que representan adquisiciones "pacíficas" del Vaticano II: la sacramentalidad del matrimonio, la dignidad de los esposos, el amor conyugal y "la función de los elementos afectivos" en la relación de pareja. Según Z. Herrero, la positiva valoración de la sexualidad que hace GS resulta muy beneficiosa para la moral matrimonial porque la unidad e igualdad de los "compartes" no sólo son imperativos sociales, sino, sobre todo, exigencias del amor.

También encontramos un estudio sobre GS perteneciente al período de los años 80, en el que apenas hay un par de artículos en EstAg acerca de la doctrina conciliar. Es un texto breve del sacerdote y periodista valenciano Antonio Díaz-Tortajada. Contiene una reflexión filosófico-teológica

19 Ib. 94; cf. Ib. 98-99. GS 10: "En realidad los desequilibrios que sufre el mundo moderno están relacionados con aquel otro desequilibrio más fundamental que tiene sus raíces en el corazón del hombre".

20 Cf. Pablo VI, Homilía Summi Pontificis (7 de diciembre de 1965): Acta Synodalia IV,VII, 662.

21 Z. HERRERo Bravo, "Algunos puntos conciliares en torno a la doctrina del matrimonio": EstAg 2 (1967) 319-346. 
sobre la Exposición preliminar de la Constitución pastoral: “La condición del hombre en el mundo de hoy" (GS 4-10)22. El estudio, dividido en cinco apartados, hace un análisis de la realidad en que se sitúa el documento conciliar estudiado desde varias perspectivas: antropológica, metafísica, sociológica, etc. En el comentario de los textos de GS, sobre todo, GS 10 y GS 21-22, el autor recurre abundantemente al pensamiento de los grandes filósofos modernos (Kant, Hegel) y contemporáneos (Marx, Nietzsche, Heidegger, Scheler, Wittgenstein, Rilke, Bloch) -que cita expresamente-, así como de algunos teólogos (J. Moltmann, J. B. Metz).

Los dos trabajos más recientes sobre GS aparecidos en EstAg son del comienzo de presente siglo. Ambos tienen como autor a Fernando Bogónez, doctor en Teología y profesor de la Universidad de Valladolid. El primero de ellos, titulado "La persona humana en su relación con Dios"23, reproduce la primera parte de su tesina de Licenciatura en Teología sobre la persona humana en GS (1999) ${ }^{24}$. La propuesta antropológica de GS considera indispensable conocer la relación del hombre con Dios para poder comprender al propio hombre. El artículo consta de tres partes. Siempre al hilo de la enseñanza de GS, en la primera, "Dios al encuentro del hombre", el autor se interroga sobre el porqué de la relación primigenia entre Dios y el hombre. La segunda parte, "La persona humana creada a imagen de Dios", trata la relación del hombre como criatura con Dios; su grandeza y su miseria. Por último, en la tercera parte, "La filiación divina en Cristo", se señala cómo Cristo, mediante su encarnación y redención, ha renovado el vínculo de unión entre Dios y el hombre. En esa última parte hay un estudio exhaustivo de GS 22 ("Cristo, el hombre nuevo") en el que se comentan minuciosamente a cada uno de los seis párrafos de ese destacado texto de la Constitución pastoral -para muchos comentaristas el más relevante del Vaticano II desde el punto de vista cristológico-. "El deseo de lo absoluto, que nace de lo profundo del hombre, sólo lo puede llenar Dios, porque es el origen y meta del hombre" 25 , concluye el autor, evocando la célebre expresión agustiniana (cf. Conf. 1,1,1), que cita GS 21g.

22 A. Díaz-TorTAJADA, "La condición del hombre en el mundo actual. Cuadro de referencias para una lectura de la exposición preliminar de la Gaudium et Spes": EstAg 18 . (1983) 81-97.

23 F. Bogónez Herreras, "La persona humana en su relación con Dios según la Constitución pastoral Gaudium et spes": EstAg 35 (2000) 533-573.

24 ID., La persona humana como ser relacional según la "Gaudium et spes", Burgos 1999, pp. 46-127. En su tesis doctoral (Burgos 2004) el autor profundiza y desarrolla este mismo tema desde las perspectivas histórica y teológica.

25 ID., "La persona humana en su relación con Dios", 571. 
El segundo artículo del profesor Bogónez es un estudio monográfico sobre la noción de "mundo" en GS"6. El trabajo se desarrolla en cuatro apartados. El primero, de carácter introductorio ("Enfoque previo"), sitúa el objeto de estudio en el contexto de la temática propia de la Constitución pastoral: la preocupación de la Iglesia por el mundo. En el segundo apartado se presenta la "concepción material y sociológica" del mundo que domina en las sucesivas redacciones de los dos primeros esquemas de GS (1963-1964). El tercer apartado estudia "la primera definición compleja" del término mundo en el esquema debatido durante el cuarto período de sesiones (1965), conocido como "esquema de Ariccia". En este texto se ponen de relieve los aspectos cosmológico, antropológico y soteriológico del mundo. En el apartado final el autor destaca la perspectiva teológica que comprende el mundo como "familia humana". Ese enfoque teológico de fondo redimensiona los otros aspectos de la noción de mundo: antropológico y material, histórico y dinámico, soteriológico y escatológico. Al final de su ensayo el autor propone cuatro conclusiones para "seguir reflexionando". Las dos primeras son de índole general: el carácter antropológico del mundo de GS y la recta comprensión de la justa autonomía de las realidades terrenas (que "no significa que Dios sobre en la historia de la humanidad", cf. GS 36). Las últimas conclusiones tienen que ver con el contexto (europeo) occidental en particular: la Iglesia se encuentra ahí en "estado de misión" (nueva evangelización) y una antropología sin Dios es fuente de desesperanza (cf. Ecclesia in Europa $\mathrm{n.9)}$.

\section{ESTUDIOS SOBRE OTROS DOCUMENTOS CONCILIARES.}

Hay solamente tres trabajos que tienen como objeto el estudio particularizado de otros documentos del Vaticano II. Curiosamente los tres tienen como tema la Vida Consagrada, si bien la estudian desde diversos ángulos y no en un único documento. Como es sabido, además de ser el objeto del decreto PC, la vida religiosa tiene un importante espacio en el cap. VI de la Constitución sobre la Iglesia (cf. LG 43-47). De los tres estudios, el más antiguo en el tiempo (Tarsicio Jáñez) trata, en general, de $l a$ vocación religiosa en el Vaticano $I I^{27}$. Está dividido en tres partes. La pri-

26 ID., "El mundo comprendido como familia humana y su dimensión teológica: la aportación de Gaudium et Spes 2b": EstAg 40 (2005) 305-327.

27 T. JÁÑEZ BARRIO, "La vocación religiosa en el Vaticano II": EstAg 2 (1967) 369-383. 
mera parte es la crónica de la "IV Asamblea Nacional de Delegados y Delegadas de Vocaciones Religiosas". Un encuentro organizado por el Secretariado de Vocaciones de CONFER con el tema: "La Pastoral de las vocaciones a la luz del Vaticano II". Por consiguiente, se abordó esa problemática de la vida religiosa desde el punto de vista pastoral. Ahora bien, como indica el autor, la enseñanza del Vaticano II iluminó las exposiciones "tanto en lo doctrinal, como en lo práctico". El autor destaca, entre otras, las siguientes ponencias: "La prioridad de la vocación bautismal" (J. Martínez Vega, O.M.I), "Vida y vocación religiosas" (C. Godoy, F.S,C.), "El obispo y la pastoral de las vocaciones" (Mons. A. Añoveros, obispo de Cádiz) y "La vocación misionera a la luz del Concilio" (J. Mª Boiburu). En su segunda parte, este artículo contiene un estudio sobre la "Teología de la vocación". Y la tercera parte trata la amplia temática de la "Pastoral vocacional". El artículo culmina enumerando diez "puntos de reflexión".

Los últimos artículos que reseñamos son sendos estudios de espiritualidad de la Vida Religiosa. En un largo artículo -separado en dos entregas por su extensión ${ }^{28}$ - el profesor Heliodoro Andrés, agustino y docente de derecho canónico en el Estudio Teológico de Valladolid (19671976), analiza la problemática del voto de obediencia a partir de PC 14. El Concilio Vaticano II indica cómo llevar a la práctica la concepción evangélica de la relación autoridad-obediencia. Al hilo de la enseñanza conciliar, el estudio se desarrolla en cuatro partes. La primera parte -la más larga- presenta las "Consideraciones previas" y las cuatro primeras disposiciones -"exigencias", dice el autor-que reclama el Concilio a los superiores religiosos en el ejercicio de la autoridad: responsabilidad, docilidad, servicio y amor. La segunda entrega del artículo comprende las otras tres partes del trabajo. Se completa la enumeración de las cualidades del superior con el respeto a la persona y la libertad (segunda parte) y con la colaboración, el diálogo y el sentido de la autoridad (tercera parte). Por último, en la cuarta parte, el autor identifica la representatividad, la fidelidad, la reverencia y la sinceridad como características fundamentales de los órganos colectivos de gobierno ("Capítulos y Consejos").

$M^{a}$ Ascensión Matás, dominica, también profesora del Estudio Teológico, firma el otro artículo. Se trata de un trabajo presentado como tesina de Licenciatura, que tiene como tema la misión de la vida religiosa ${ }^{29}$. Es

28 H. ANDRÉs PUENTE, "Ejercicio de la autoridad (Decreto "Perfectae Caritatis", $\mathrm{n}^{\circ}$ 14)": EstAg 3 (1968) 3-54; 219-317.

${ }^{29} \mathrm{M}^{\mathrm{a}}$ A. MATÁs GARCíA, "El desarrollo de la misión de la vida religiosa durante la época del concilio Vaticano II y el período posconciliar": EstAg 46 (2011) 33-67. 
puramente casual que se haya dejado precisamente para el final la reseña del único texto sobre el Vaticano II aparecido en EstAg que tiene a una mujer por autora. Es un estudio diacrónico del magisterio conciliar acerca del tema y de su recepción postconciliar. Recorre la segunda mitad del s. XX en cuatro secciones, correspondiendo cada una de ellas a una década del siglo pasado. En la primera sección (años 60), además de presentar el análisis de los textos conciliares (LG, PC), la autora estudia también otros documentos pontificios que tocan la cuestión como la Encíclica Ecclesiam Suam (1964). En la segunda parte (años 70) se estudia el papel de los religiosos en la tarea evangelizadora según el magisterio de las Exhortaciones Apostólicas Evangelica Testificatio (1971) o Evangelii Nuntiandi (1976) y su posición dentro de la Iglesia en el documento "Mutuae Relationes" (1978). De los años 80, destacan las aportaciones de los documentos "Dimensión contemplativa de la vida consagrada" (1980) y "Religiosos y promoción humana" (1980), producidos por la Sagrada Congregación para los Institutos de Vida Religiosa y las Sociedades de Vida Apostólica. Finalmente, los documentos más actuales -y seguramente, también, los más relevantes sobre la cuestión- son los publicados en el último decenio del s. XX: las "Orientaciones sobre la formación en los institutos religiosos" (1990), la Exhortación Apostólica Vita Consecrata (1996) y las instrucciones "La vida fraterna en comunidad" (1994) y "Caminar desde Cristo" (2002). Este último documento publicado ya en el presente siglo. En la conclusión, la autora afirma que la misión de la vida religiosa no es otra que la misma misión de la Iglesia. Y esa misión consiste fundamentalmente en "autotrascenderse dentro de la historia y por la historia, como memoria incitante hacia un futuro de fidelidad creadora (cf. VC 37), que transforma la realidad y se deja trasformar por ella".

\section{CONCLUSIÓN}

Al final de este breve recorrido por los textos que estudian el Vaticano II en las páginas de EstAg podemos sacar alguna conclusión. Por un lado, hay que reconocer que los estudios aparecidos sobre el tema son muy minoritarios dentro del conjunto de los -varios centenares- publicados en los 47 números de la revista. Si bien es cierto que la temática del Vaticano II suscitó una proliferación de estudios en la primera década de la publicación, el interés decae después casi totalmente hasta el nuevo siglo. Con la sola excepción de GS, los artículos aparecidos no prestan una especial atención a ningún documento conciliar en particular. Sin embar- 
go, por otra parte, es justo valorar la riqueza de los enfoques, la talla intelectual de muchos de los autores -la mayoría de los cuales son agustinosy el buen nivel general de los estudios reseñados. Es más, algunos de ellos sobresalen por su calidad y han sido citados a menudo en trabajos posteriores sobre la materia que tratan.

La presente reseña aparece en un volumen que rinde homenaje al Concilio Vaticano II con motivo de su cincuentenario. Deseamos que inaugure también una nueva época de aportaciones de esta revista al estudio y difusión de las enseñanzas del Vaticano II. 
